\title{
ADRB2 polymorphism Arg16Gly modifies the natural outcome of heart failure and dictates therapeutic response to $\beta$-blockers in patients with heart failure
}

\author{
Jin Huang ${ }^{1,2}$, Chenze Li ${ }^{1,2}$, Ying Song ${ }^{3}$, Xiaohan Fan ${ }^{4}$, Ling You', Lun Tan', Lei Xiao', Qing Li', Guoran Ruan', \\ Senlin $\mathrm{Hu}^{1}$, Wei Cui ${ }^{5}$, Zongzhe $\mathrm{Li}^{1}$, Li Ni ${ }^{1}$, Chen Chen ${ }^{1,2}$, Anthony Yiu-Ho Woo ${ }^{3,6}$, Rui-Ping Xiao ${ }^{3}$ and Dao Wen Wang ${ }^{1,2}$
}

\begin{abstract}
We sought to investigate the association of single nucleotide polymorphisms (SNPS) of the genes involved in $\beta A R$ signaling with the response of patients to $\beta A R$ blockers. A total of 2403 hospitalized patients with chronic heart failure (HF) were enrolled in a multicenter observational study as the first cohort and followed up for a mean period of 20 months. Genes for $\beta 1 A R, \beta 2 A R$, and the major cardiac G-protein-coupled receptor kinases (GRKs) GRK2 and GRK5 were analyzed to identify SNPs, and patients were stratified according to genotypes. A second independent cohort enrolling 919 patients with chronic HF was applied to validate the observed associations. The signaling properties of the key identified SNPs were assessed in vitro. Our data showed that HF patients harboring the Gly 16 allele in the gene for $\beta 2 A R$ (ADRB2) had an increased risk of the composite end point relative to patients who were homozygous for Arg16. Notably, these patients showed a beneficial response to $\beta A R$-blocker treatment in a $G$ allele-dose-dependent manner, whereas Arg16 homozygotes had no response to $\beta A R$-blocker therapy. This Arg16Gly genotype-dependent heterogeneity in clinical outcomes of HF was successfully validated in the second independent population. Besides, the in vitro experiments revealed that $\mathrm{G}$ allele carriers were defective in $\beta 2 A R$-coupled inhibitory adenylate cyclase $\mathrm{g}$ $\left(\mathrm{G}_{\mathrm{i}}\right)$ protein signaling.
\end{abstract}

\section{Introduction}

There is currently a global epidemic of heart failure (HF), with over 915,000 newly diagnosed patients with HF each year in the United States ${ }^{1}$. This complex syndrome is characterized by an interplay among genetic, neurohormonal, inflammatory, and metabolic factors ${ }^{2,3}$. HF therapy has been improved greatly over the past two

\footnotetext{
Correspondence: Rui-Ping Xiao (xiaor@pku.edu.cn) or

Dao Wen Wang (dwwang@tjh.tjmu.edu.cn)

'Division of Cardiology, Departments of Internal Medicine, Tongji Hospital, Tongji Medical College, Huazhong University of Science \& Technology, 430030 Wuhan, China

${ }^{2}$ Hubei Key Laboratory of Genetics and Molecular Mechanisms of Cardiologic Disorders, 430030 Wuhan, China

Full list of author information is available at the end of the article.

These authors contributed equally: Jin Huang, Chenze Li, Ying Song
}

decades, particularly with the use of angiotensinconverting enzyme inhibitors and $\beta 1$-adrenergic receptor ( $\beta 1 \mathrm{AR})$ antagonists, which inhibit pathological remodeling and apoptosis of cardiomyocytes ${ }^{4,5}$. The current American College of Cardiology (ACC)/American Heart Association (AHA) guidelines recommend nondiscriminatory prescription of $\beta$-blockers to all stable HF with reduced ejection fraction (HFrEF), except for patients with comorbid contradictory conditions or who are unable to tolerate treatment with these drugs ${ }^{6}$. However, a substantial proportion of HF patients receive little or no benefit from the available therapeutics-often specifically owing to a lack of response to $\beta$-blockers ${ }^{7-10}$ and 5 -year mortality is still $>50 \%{ }^{1}$. Changing official guidelines to include recommendations for patient 
stratification in the management of HF may open new avenues for the development of more effective and personalized therapies. For instance, new ways of identifying poor $\beta$ AR-blocker responders before commencing treatment may revolutionize clinical practice and improve health management of HF patients.

During the progression of $\mathrm{HF}$, the sympathetic nervous system becomes hyperactive. The resultant increase in $\beta$-AR stimulation to cardiomyocytes initially produces a positive inotropic effect, primarily via the activation of the $\beta 1 \mathrm{AR}-$ stimulating adenylate cyclase $\mathrm{g}$ (Gs) protein -adenylyl cyclase-cyclic adenosine monophosphate (cAMP)-protein kinase A signaling cascade ${ }^{11}$. However, persistent $\beta 1$ AR stimulation triggers apoptosis of cardiomyocytes and leads to hypertrophy, fibrosis and maladaptive remodeling of the diseased hearts, via mechanisms that depend on calcium/ calmodulin-dependent kinase type II (CaMKII), but not on protein kinase $\mathrm{A}^{12,13}$.

$\beta 1 A R$ is the predominant $\beta A R$ subtype in the heart and the major mediator of the positive inotropic action of catecholamines under physiological conditions. During $\mathrm{HF}$, however, $\beta 1 \mathrm{AR}$ is markedly desensitized and its expression is selectively downregulated. Consequently, the $\beta 1 A R: \beta 2 A R$ ratio decreases from 4:1 in the normal heart to $3: 2$ in the failing heart, making the role of the $\beta 2 A R$ subtype more important in the failing heart ${ }^{14}$. In contrast to $\beta 1 A R$ that couples only to $G_{s}$ proteins, $\beta 2 A R$ couples to both $G_{s}$ and inhibitory adenylate cyclase $g\left(G_{i}\right)$ proteins ${ }^{15}$. $\beta 2 A R$, therefore, has a distinct role in the heart. This becomes especially relevant under the pathological conditions of $\mathrm{HF}$ in which the expression of $G_{i}$ proteins is elevated in the absence of alteration in the expression of $G_{s}$ proteins ${ }^{16,17}$. Specifically, $\beta 2 A R-G s$ mediated contractile support in failing heart while the activation of $\beta 2 A R$-coupled $G_{i}$ signaling protects the heart against various insults-induced loss of cardiomyocytes via the $\mathrm{G}_{\mathrm{i}}-\mathrm{G} \beta \gamma$-phosphatidylinositol 3-kinase (PI3K)-AKT cell survival pathway ${ }^{18,19}$.

It is unclear how genetic factors contribute to $\beta 2 A R$ signaling pathway and/or the differences in response to $\beta$ blockers in HF patients. To address this important question, we genotyped key genes involved in the $\beta A R$ signaling pathway-ADRB1, ADRB2, GRK2, and GRK5in blood samples from patients with HF and healthy control individuals. We then assessed the relationship between the identified gene variants and the prognosis of HF in a large cohort of patients with or without $\beta$-blocker treatment. In vitro experiments were designed to assess the signaling properties of the key identified variants.

\section{Results}

\section{Resequencing data}

By resequencing $A D R B 1, A D R B 2, G R K 2$, and GRK5 genes in blood samples from 100 pairs of patients with HF and healthy individuals, five missense variants- $A D R B 1$ Arg389Gly C $>\mathrm{G}, A D R B 2$ Arg16Gly A $>\mathrm{G}, A D R B 2$ Gln27Glu C > G, GRK5 Gln41Leu $\mathrm{A}>\mathrm{T}$, and GRK5 $\operatorname{Arg} 304 \mathrm{His} \mathrm{G}>\mathrm{A}$-and six synonymous variants were detected in the candidate genes (Supplementary Table S1); none of these single nucleotide polymorphism (SNPs) were located within GRK2. For all 11 SNPs identified, the minor allele frequency did not differ significantly between the HF and control groups, suggesting that the polymorphisms were not associated with the risk of HF.

\section{Patients and follow-up}

A total of $2615 \mathrm{HF}$ patients were recruited between 1 January 2009 and 31 October 2014 in the first cohort. Of the patients recruited, 212 were excluded from enrollment owing to repeat admittance (for 149 patients; $5.7 \%$ of all recruited) or valvular heart disease (in 63 patients; $2.4 \%$ of all recruited). Accordingly, 2403 patients were genotyped (Fig. 1). Among the overall study population (mean age $59.3 \pm 14.5$ years, $66.6 \%$ male), $56.6 \%$ had severe cardiac symptoms (New York Heart Association, (NYHA) class III or IV); $87.5 \%$ had a history of hypertension; $24.8 \%$ had a history of hyperlipidemia; and $31.8 \%$ had diabetes (Table 1). When study participants were stratified by genotype of the ADRB2 Arg16Gly A > G locus (AA, AG, and GG), baseline characteristics did not differ significantly among the three groups (Table 1), except for hypertension, which was slightly less prevalent among patients with the AA genotype (84.4\% for AA genotype group, $89.6 \%$ for the AG genotype group, and $89.1 \%$ for the GG genotype group; $P<0.001$ ).

At the last assessment, the study follow-up compliance rate was $99.2 \%(2386 / 2403)$, and the mean follow-up period was 20.3 months (maximum: 60 months), with no significant difference among genotype groups (21.2, 19.6 and, 20.1 months for the AA, AG, and GG groups, respectively, $P=0.362$ ). During follow-up, $57.2 \%$ of patients included in the outcome analysis were on $\beta$ ARblocker therapy (metoprolol or bisoprolol) for $\geq 6$ months.

The basic characteristics of the patients in the replicated cohort were summarized in Supplementary Table S2 and the mean follow-up time was 15 months in the second cohort.

\section{Study outcomes for entire cohort in the first cohort}

At the July 2015 cutoff date for the first cohort, cardiovascular death or heart transplantation (the primary end point) had occurred in 419 patients (17.6\%). During follow-up, 448 (18.8\%) patients died and 391 (16.4\%) of them died from cardiovascular causes. Rehospitalization occurred in 929 (38.9\%) patients, with 708 (29.7\%) being rehospitalized for cardiovascular reasons. The recurrence of HF was observed in 628 (26.3\%) patients, and 40 (1.7\%) 


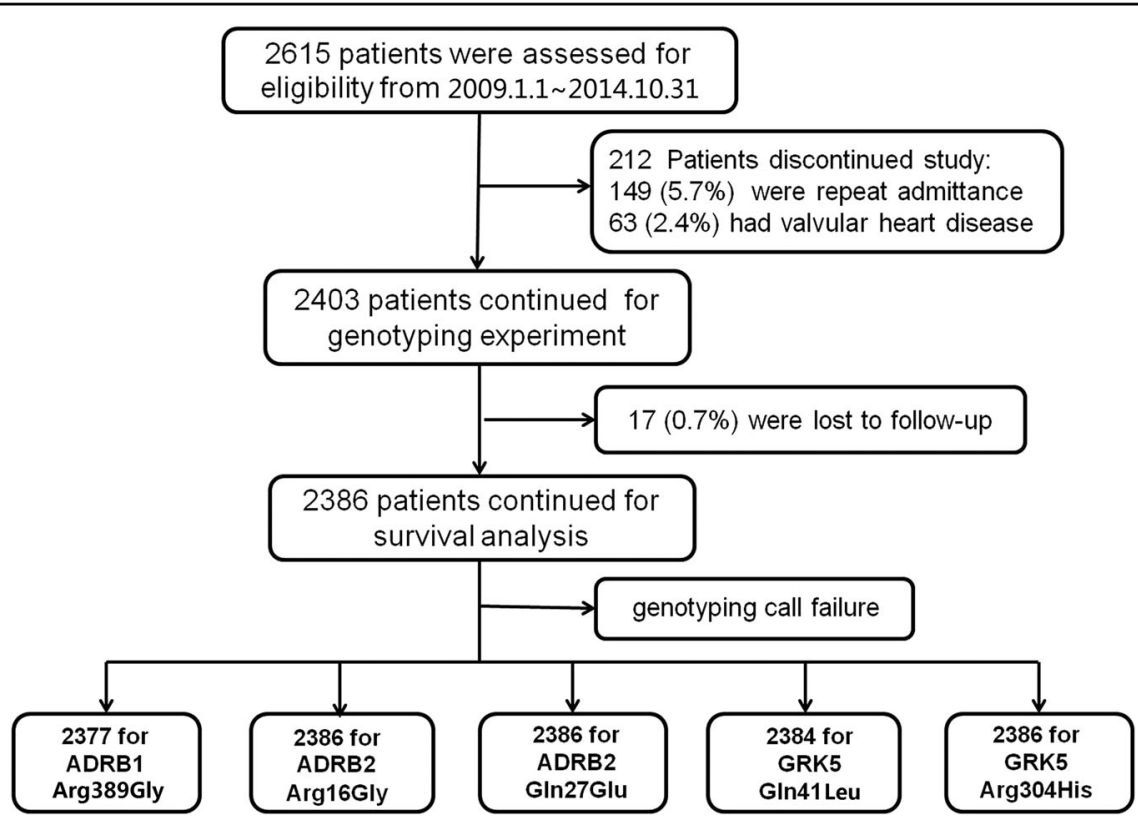

Fig. 1 Enrollment and follow-up of patients with HF in the first stage. At study termination on July 2015 in the first discovery stage, 2615 patients with HF had been screened for eligibility to be included in the study in three centers in China and 2403 patients were finally enrolled and genotyped. Of these patients, 149 (5.7\%) were excluded for repeat admittance, and 63 (2.4\%) were excluded because valvular heart disease was considered the main reason for HF. Of the study participants, 17 were lost from follow-up because incorrect contact information had been provided on enrollment. Of the 2386 patients included in the outcome analysis: all were included in the analysis of the prognostic utility of the ADRB2 Arg16Gly, ADRB2 Gln27Glu, and GRK5 Arg304His variants; 2377 patients were included in the analysis of the prognostic utility of the ADRB1 Arg389Gly variants; and 2384 patients were included in the analysis of the prognostic utility of the GRK5 GIn41Leu variants

patients experienced new onset of stroke. The impact of conventional clinical risk factors on HF prognosis was assessed for the study cohort (Supplementary Table S3). Increasing age (hazard ratio (HR), 1.02; $95 \%$ confidence interval (CI), $1.01-1.03 ; P<0.001)$, a history of diabetes (HR, 1.53; 95\% CI, 1.26-1.86; $P<0.001$ ), the etiology of the HF (nonischemic versus ischemic; $\mathrm{HR}, 1.28$; $95 \% \mathrm{CI}$, $1.05-1.56 ; P=0.013$ ), NYHA functional class (class III + IV versus class II; HR, 3.04; 95\% CI, 2.39-3.87; $P<0.001$ ), and the level of serum NT-proBNP (HR, 1.55; 95\% CI, $1.45-1.67 ; P<0.001)$ were significantly and positively associated with the risk of the primary end point in our study.

Of the five missense variants we identified in the exons of the ADRB1, ADRB2, and GRK5 genes, only ADRB2 Arg16Gly A > G (G being the minor allele, with a frequency of $38.2 \%$ in our HF cohort) was significantly associated with heterogeneity in the primary end point and an individual end point of cardiovascular deaths (both $P<0.001$ by Log rank test) (Fig. 2a, b). The primary end point-cardiovascular death or heart transplantationoccurred in 129 (14.0\%) patients with the AA genotype, compared with 209 (18.8\%) patients with the AG genotype (HR, 1.42; 95\% CI, 1.14-1.77; $P=0.002$ for AG versus $\mathrm{AA}$ ) and 81 (22.8\%) patients with the GG genotype (HR, 1.22; 95\% CI, $0.94-1.57 ; P=0.136$ for GG versus
AG and HR, 1.71; 95\% CI, 1.30-2.26; $P<0.001$ for GG versus $A A$, respectively). By combining the patients with AG and GG genotypes into a single group, we found that the patients carrying $\mathrm{G}$ allele had a $50 \%$ higher risk of cardiovascular death or heart transplantation (HR, 1.49; 95\% CI, 1.21-1.83; $P<0.001$ for AG/GG versus AA; Table 2). However, when the analyses were conducted on patient subgroups with reduced or preserved left ventricular ejection fraction, the genotype-dependent variability in prognosis was only detected in the subgroup of HFpEF (heart failure with preserved ejection fraction) but not in the HFrEF subgroup (Supplementary Fig. S1).

During follow-up, 15.0\% (138/919) of patients in the AA group, 20.3\% (226/1112) of patients in the AG group, and $23.7 \%(84 / 355)$ of patients in the GG group died (HR, 1.50; $95 \%$ CI, 1.22-1.83; $P<0.001$ for AG/GG versus AA; Table 2). Most of these deaths (124 in the AA group, 193 in the AG group, and 74 in the GG group) had cardiovascular causes and the $G$ allele was associated with increased risk of this individual end point (HR, 1.44; 95\% CI, $1.16-1.79 ; P=0.001$ for AG/GG versus AA; Table 2). The heterogeneity in all-cause mortality, rehospitalization rate and improvement in NYHA class were similarly associated with the genotypes of this SNP. The recurrence of HF did not differ among the three genotype groups (Table 2). 
Table 1 Clinical characteristics of the patients by ADRB2 Arg16Gly A > genotype

\begin{tabular}{|c|c|c|c|c|c|}
\hline Characteristics & $\begin{array}{l}\text { All patients } \\
(N=2403)\end{array}$ & $\begin{array}{l}\text { Patients with AA } \\
\text { genotype } \\
(N=923)\end{array}$ & $\begin{array}{l}\text { Patients with AG } \\
\text { genotype } \\
(N=1122)\end{array}$ & $\begin{array}{l}\text { Patients with GG } \\
\text { genotype } \\
(N=358)\end{array}$ & $P$ value $^{\mathrm{a}}$ \\
\hline Age (years) & $59.3 \pm 14.5$ & $58.7 \pm 14.5$ & $59.0 \pm 14.6$ & $59.8 \pm 14.5$ & 0.208 \\
\hline Male sex ${ }^{b}$ & $1601(66.6)$ & $616(66.7)$ & $752(67.0)$ & $233(65.1)$ & 0.791 \\
\hline Ischemic etiology ${ }^{\mathrm{b}}$ & $1137(47.3)$ & $450(48.8)$ & $525(46.8)$ & $162(45.3)$ & 0.343 \\
\hline \multicolumn{6}{|l|}{ NYHA functional class ${ }^{\mathrm{b}}$} \\
\hline$\|$ & $1042(43.4)$ & $417(45.2)$ & $479(42.7)$ & $146(40.8)$ & \\
\hline III & $812(33.8)$ & $316(34.2)$ & 379 (33.8) & $117(32.7)$ & 0.201 \\
\hline IV & $549(22.8)$ & $190(20.6)$ & $264(23.5)$ & $95(26.5)$ & \\
\hline \multicolumn{6}{|l|}{ Personal history ${ }^{\mathrm{b}}$} \\
\hline Smoking & $937(39.0)$ & $365(39.5)$ & $436(38.9)$ & $136(38.0)$ & 0.87 \\
\hline Hypertension & $2103(87.5)$ & $779(84.4)$ & 1005 (89.6) & $309(89.1)$ & $<0.001$ \\
\hline Hyperlipidemia & $597(24.8)$ & $241(26.1)$ & $265(23.6)$ & $91(25.4)$ & 0.415 \\
\hline Diabetes & $765(31.8)$ & $290(31.4)$ & $353(31.5)$ & $122(34.1)$ & 0.614 \\
\hline Stroke & $246(10.2)$ & $102(11.1)$ & $113(10.1)$ & $31(8.7)$ & 0.434 \\
\hline \multicolumn{6}{|l|}{ Clinical testing } \\
\hline Systolic pressure $(\mathrm{mm} \mathrm{Hg})$ & $131.2 \pm 25.2$ & $131.8 \pm 25.2$ & $129.0 \pm 24.2$ & $131.3 \pm 25.5$ & 0.243 \\
\hline Diastolic pressure $(\mathrm{mm} \mathrm{Hg})$ & $80.3 \pm 16.2$ & $80.7 \pm 16.8$ & $78.6 \pm 14.4$ & $80.5 \pm 16.2$ & 0.1 \\
\hline Heart rate (beats/min) & $83.2 \pm 20.2$ & $82.7 \pm 20.0$ & $83.5 \pm 20.0$ & $83.6 \pm 20.4$ & 0.573 \\
\hline Serum creatinine $(\mu \mathrm{mol} / /)^{c}$ & $84(67-106)$ & $82(66-105)$ & $84(69-107)$ & $84(66-107)$ & 0.232 \\
\hline NT-proBNP $(p g / m l)^{c}$ & $1857(424-5652)$ & $1859(514-5013)$ & $1774(405-5706)$ & $2131(351-6962)$ & 0.435 \\
\hline LVEDD $(\mathrm{mm})^{c}$ & $55(48-64)$ & $54(47-63)$ & $55(48-64)$ & $56(47-64)$ & 0.152 \\
\hline $\operatorname{LAD}(\mathrm{mm})^{c}$ & $40(35-45)$ & $39(34-45)$ & $40(35-45)$ & $40(36-46)$ & 0.2 \\
\hline Ejection fraction $(\%)^{c}$ & $47(33-61)$ & $48(33-62)$ & $47(32-60)$ & $44(32-61)$ & 0.334 \\
\hline \multicolumn{6}{|l|}{ Medication (\%) } \\
\hline Digoxin & 563 (23.6) & $214(23.3)$ & $274(24.6)$ & $75(21.1)$ & 0.382 \\
\hline Diuretics & $1104(46.3)$ & $402(43.7)$ & $527(47.4)$ & $175(49.3)$ & 0.121 \\
\hline ACEI & $1246(52.2)$ & $466(50.7)$ & $584(52.5)$ & $196(55.2)$ & 0.340 \\
\hline ARB & $279(11.7)$ & $102(11.1)$ & $142(12.8)$ & $35(9.9)$ & 0.257 \\
\hline Beta-blocker & $1365(57.2)$ & $534(58.1)$ & $636(57.2)$ & 195 (54.9) & 0.590 \\
\hline Spironolactone & 938 (39.3) & $354(38.5)$ & 437 (39.3) & $147(41.4)$ & 0.639 \\
\hline
\end{tabular}

$A C E l$ angiotensin-converting enzyme inhibitor, $A R B$ angiotensin receptor blockers, $L A D$ left atrial dimension, $L V E D D$ left ventricular end-diastolic dimension, NT-proBNP the N-terminal pro-hormone of brain natriuretic peptide, NYHA New York Heart Association

${ }^{a}$ For similarity among the different genotypes

${ }^{b}$ Listed as number (\%)

Interquartile range included in parentheses

Among the other four missense variants in the ADRB1, $A D R B 2$, and GRK5 genes, only the AT genotype of GRK5 Gln41Leu (A > T, with a minor allele frequency of $1.2 \%$ in our study) was associated with an altered risk of any of the end points assessed. Patients with the AT genotype of
GRK5 Gln41Leu had a lower rate of cardiovascular rehospitalization $(29.9 \%$ in the AA group versus $18.6 \%$ in the AT group, $P=0.029$; no one in our cohort had the TT genotype) rather than the primary end point and all-cause death (Supplementary Table S4). 


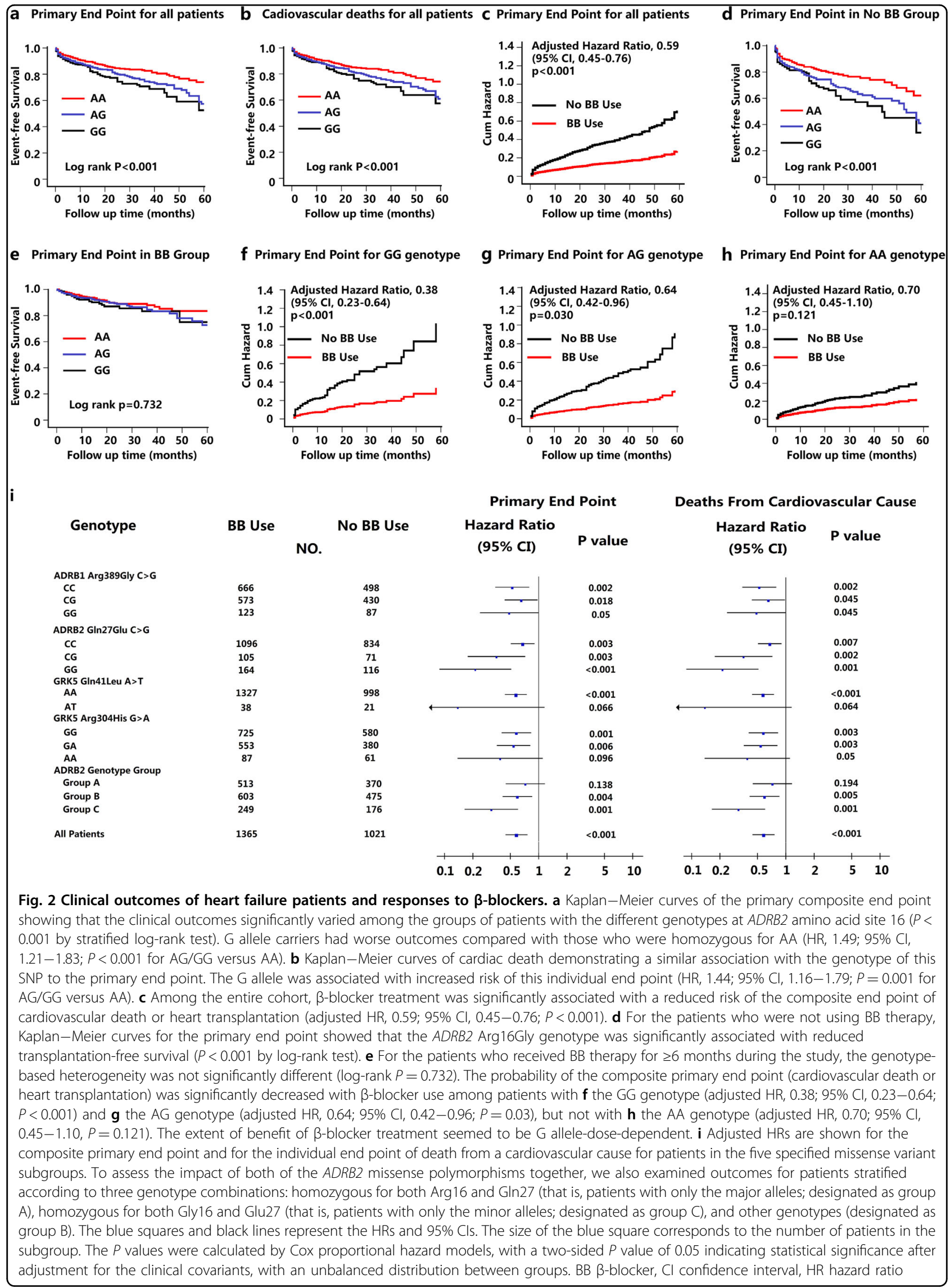


Table 2 Primary, secondary, and other related outcomes, according to ADRB2 Arg16Gly (A > G)

\begin{tabular}{|c|c|c|c|c|c|}
\hline Outcome & $\begin{array}{l}\text { AA genotype } \\
(N=919)\end{array}$ & $\begin{array}{l}\text { AG genotype } \\
(N=1112)\end{array}$ & $\begin{array}{l}\text { GG genotype } \\
(N=355)\end{array}$ & $\begin{array}{l}\mathrm{HR}^{\mathrm{a}}(95 \% \mathrm{Cl}) \text { or } \mathrm{OR}^{\mathrm{b}} \\
\mathrm{AG} / \mathrm{GG} \text { vs. } \mathrm{AA}\end{array}$ & $P$ value \\
\hline \multicolumn{6}{|l|}{ Primary composite outcome-no. (\%) } \\
\hline Death from cardiovascular causes or heart transplantation & $129(14.0)$ & $209(18.8)$ & $81(22.8)$ & $1.49(1.21-1.83)$ & $<0.001$ \\
\hline Death from cardiovascular causes & $124(13.5)$ & $193(17.4)$ & $74(20.8)$ & $1.44(1.16-1.79)$ & 0.001 \\
\hline \multicolumn{6}{|l|}{ Secondary outcomes—no. (\%) } \\
\hline Death from any cause & $138(15.0)$ & $226(20.3)$ & $84(23.7)$ & $1.50(1.22-1.83)$ & $<0.001$ \\
\hline First hospitalization for cardiovascular causes & $243(26.4)$ & $355(31.9)$ & $110(31.0)$ & $1.26(1.08-1.48)$ & 0.003 \\
\hline First hospitalization for any cause & $317(34.1)$ & $482(43.3)$ & $130(36.6)$ & $1.30(1.13-1.49)$ & $<0.001$ \\
\hline Recurrence of heart failure & $228(24.8)$ & $304(27.3)$ & $99(27.9)$ & $1.15(0.98-1.36)$ & 0.086 \\
\hline \multicolumn{6}{|l|}{ Other related outcomes—no. (\%) } \\
\hline New onset of stroke & $12(1.3)$ & $23(2.1)$ & $5(1.4)$ & $1.41(0.72-2.78)$ & 0.320 \\
\hline Improvement in NYHA class & $505(55.0)$ & $557(50.1)$ & $170(47.9)$ & $1.22(1.03-1.45)$ & 0.021 \\
\hline
\end{tabular}

NYHA New York Heart Association

${ }^{a} \mathrm{HR}$, hazard ratios and $P$ value were calculated with the use of stratified Cox proportional hazard models adjusting with gender, age, and history of hypertension

${ }^{b}$ Improvement in heart function was analyzed as a binary outcome with the use of logistic regression model to calculate the odds ratio and $P$ value

\section{Effects of $\beta$-blocker treatment on the prognosis of heart} failure

Consistent with findings from previous clinical trials ${ }^{20-22}$, $\beta$ AR-blocker therapy was associated with a reduced risk of the composite primary end point (adjusted HR, 0.59; 95\% CI, $0.45-0.76 ; P<0.001)$; cardiovascular death or heart transplantation occurred in $10.9 \%(149 / 1365)$ of patients taking $\beta A R-b l o c k e r$ therapy and $26.4 \%$ (270/1021) of patients who were naïve to $\beta$-blockers (Fig. 2c). The risk of the individual end point of death from a cardiovascular cause was similarly reduced among patients taking $\beta A R$ blockers (adjusted HR, 0.58; 95\% CI, 0.44-0.76; $P<0.001$ ) (Supplementary Fig. S2a); however, we detected no association between $\beta$ AR-blocker therapy and rate of rehospitalization owing to a cardiovascular cause in our study $(P=0.788)$ (Supplementary Fig. S2b).

In the absence of a $\beta A R$-blocker intervention, transplantation-free survival (that is, the primary end point of the study) varied significantly among the groups stratified by the ADRB2 Arg16Gly genotypes. Patients with the $G$ allele were more likely to experience the primary end point (35.6\% for patients with GG genotype, $29.8 \%$ for patients with AG genotype, and 19.4\% for the patients with AA genotype; Log rank $P<0.001$ ) (Fig. 2d). The outcome heterogeneity was not observed among the patients who were receiving $\beta A R$-blocker therapy (log-rank $P=0.732$ ) (Fig. 2e), indicating that genetic variation may influence the efficacy of $\beta$-blocker treatment.

When multivariate analysis was performed using Cox proportional hazards models, the benefits of $\beta$-blocker treatment were confirmed to vary among the different genotypes of $A D R B 2$ Arg16Gly site. A pretest on the distribution of clinical characteristics stratified by $\beta$ blocker usage and various genotypes was conducted (Supplementary Tables S5-10). Notably, for ADRB2 Arg16Gly A > G site, $\beta$ AR-blocker therapy improved the transplantation-free survival in patients with GG and AG genotypes in a $G$ allele-dose-dependent manner (adjusted HR, 0.38; 95\% CI, 0.23-0.64; $P<0.001$ in the GG genotype group; and adjusted HR, 0.64; $95 \%$ CI, $0.42-0.96 ; P=0.030$ in the AG genotype group) (Fig. 2f, g and Supplementary Fig. S3). However, after adjustments, no significant effect of $\beta A R$-blocker therapy was observed in the AA genotype subgroup (adjusted HR, 0.70; 95\% CI, 0.45-1.10; $P=0.121$; Fig. 2h, Supplementary Fig. S3). After combining AG and GG into one group, the risk of cardiovascular death or heart transplantation was decreased by approximately $50 \%$ when $\beta$-blockers were used (adjusted HR, 0.51; 95\% CI, 0.38-0.70; $P<0.001)$. Beneficial therapeutic effects of $\beta$-blocker and the $A D R B 2$ Arg16Gly site-associated heterogeneities in $\beta$-blocker responses were consistent in both HFrEF and HFpEF subgroups (Supplementary Fig. S4).

Apparent heterogeneities in the response to $\beta$-blockers were also detected for some of the other SNPs (Fig. 2i). In patients with AT genotype of the GRK5 Gln41Leu A > T site (59 patients, $2.5 \%$ of all patients) or the AA genotype of GRK5 Arg304His G > A site (148 patients, 6.2\% of all patients), $\beta$-blocker treatments were not significantly associated with a reduction in the risk of cardiovascular death or heart transplantation $(P=0.066$ and $P=0.096$, respectively), unlike in patients with the other variants at these loci. 
Although a beneficial response to $\beta$-blockers was observed in all three genotypes at the $A R D B 2$ Gln27Glu $C>G$ site, these beneficial effects were increased in a $G$ allele-dose-dependent manner (adjusted HR, 0.66; 95\% $\mathrm{CI}, 0.50-0.87 ; P=0.003$ for the $\mathrm{CC}$ group; adjusted $\mathrm{HR}$, 0.36; 95\% CI, $0.18-0.71 ; P=0.003$ for the CG group; and adjusted HR, 0.25 ; 95\% CI, $0.11-0.57 ; P<0.001$ for the GG group) (Fig. 2i). To assess the impact of both of the $A D R B 2$ missense polymorphisms together, we examined outcomes in study participants grouped on the basis of their status with regards to the two polymorphisms. The benefits associated with $\beta$ AR-blocker therapy when patients were stratified according to three genotype combinations-homozygous for both Arg16 and Gln27 (that is, patients with only the major alleles; designated as group A), homozygous for both Gly16 and Glu27 (that is, patients with only the minor alleles; designated as group $\mathrm{C}$ ), and other genotypes (designated as group B)-were analyzed. $\beta A R$-blocker treatment was associated with a significantly decreased risk of the primary end point in the patients with at least one minor allele (adjusted HR, 0.60; 95\% CI, 0.42-0.85; $P=0.004$ for genotype group $\mathrm{B}$; and adjusted HR, 0.33; 95\% CI, 0.17-0.61; $P=0.001$ for genotype group $\mathrm{C}$ ), but not in patients with no minor allele (adjusted HR 0.70; 95\% CI, 0.44-1.12; $P=0.138$ for genotype group A; Fig. 2i).

\section{ADRB2 Arg16Gly-dependent clinical outcomes in the replicated cohort}

To further substantiate our findings from the first cohort, we subsequently carried out an independent replication study by genotyping another cohort of 919 chronic HF patients to validate the observed associations. We successfully replicated the ADRB2 Arg16Gly sitedependent heterogeneity in prognosis of $\mathrm{HF}$ in this independent cohort, and the result showed that in the natural state without treatment of $\beta$-blockers, patients carrying $G$ allele (AG/GG genotype) had worse prognosis compared with patients who are AA homozygous (adjusted HR, 2.09; 95\% CI, 1.06-4.12; $P=0.034$ for AG/ GG versus AA; Supplementary Table S11). We also reinvestigated the response to $\beta A R$-blocker therapy in different genotype group according to ADRB2 Arg16Gly site. The results confirmed that, after adjusting for age, sex, NYHA functional class, and other drug treatments, G allele carriers are good responders to $\beta$-blockers (adjusted HR, 0.86; 95\% CI, 0.31-2.42; $P=0.774$ for AA genotype group; adjusted HR, 0.49; 95\% CI, 26-0.92; $P=0.026$ for AG/GG genotype group, Supplementary Table S11).

\section{Effects of $A D R B 2$ Arg16Gly variation on $\beta 2 A R$ function in vitro}

To explore the possible mechanism underlying the relationship between the $A D R B 2$ Arg16Gly polymorphism and long-term prognosis and response to $\beta$ AR-blocker therapy, we examined the downstream signaling events of different $\beta 2 \mathrm{AR}$ genotypes in primary cultured rodent myocardial cells and human peripheral lymphocytes. Transduction efficiency of the control Adeno-GFP virus was over $90 \%$ in our preliminary test on adult rat cardiomyocytes (Supplementary Fig. S5), similar to our previous reports $^{13,23}$. When rat cardiomyocytes were infected with one of the Adeno- $A D R B 2-A r g 16$, Adeno- $A D R B 2-$ Gly16 or Adeno-GFP viruses, we found successful expression of the two $\beta 2 \mathrm{AR}$ variants at comparable levels (Fig. 3a and Supplementary Fig. S6). Disruption of $G_{i}$ signaling with pertussis toxin (PTX) significantly augmented the contractile response to zinterol, a $\beta 2 A R$ agonist, in adult rat cardiomyocytes expressing human $A D R B 2-$ Arg16, but not in cells expressing ADRB2-Gly16 (Fig. 3b). Furthermore, in $A D R B 2$ knockout mouse cardiomyocytes infected with Adeno-ADRB2-Arg16 or Adeno-ADRB2-Gly16 and without PTX pretreatment, zinterol increased cell contractility to $200-300 \%$ of the basal levels (Fig. 3c, Supplementary Fig. S7). PTX treatment markedly enhanced zinterol-induced contractile response in cells expressing $A D R B 2-$ Arg16, but not in cells expressing ADRB2-Gly16 (Fig. 3c). Similar resultswere observed in lymphocytes isolated from $\mathrm{HF}$ patients with the various $\beta 2 \mathrm{AR}$ genotypes: the inhibition of $G_{i}$ signal by PTX significantly enhanced the $\beta 2 A R$ mediated elevation of cAMP levels in lymphocytes with the AA genotype $(P<0.05)$, whereas cells with AG or GG genotype were insensitive to PTX treatment (Fig. 3d). Thus, the results indicate that the ADRB2 Arg16Gly is a loss-of-function polymorphism with respect to $G_{i}$ protein-coupling in all of the used experimental systems.

\section{Discussion}

In the first cohort of this study, a multicenter observational study of 2403 patients with chronic HF reveals that only Arg16Gly $\mathrm{A}>\mathrm{G}$ polymorphism in $A D R B 2$ gene seems to influence the prognostic outcome of HF. The G allele is independently associated with a nearly 50\% increase in the risk of cardiovascular death or transplantation after HF. Furthermore, we have shown that chronic HF patients carrying the ADRB2-Gly16 allele (AG or GG genotype) have a markedly increased beneficial response to $\beta$ AR-blocker therapy in an allele-dose-dependent manner. In all, 36 and $62 \%$ reductions of risk in the composite end point of cardiovascular death or heart transplantation were observed for the AG $(P=0.03)$ and the GG genotype $(P<0.001)$, respectively (Fig. $2 \mathrm{f}, \mathrm{g})$. In contrast, after multiple adjustments, the patients with the AA genotype experienced no significant benefit from $\beta A R-b l o c k e r$ therapy (Fig. 2h). These observations can be successfully replicated in a separate chronic HF patient 


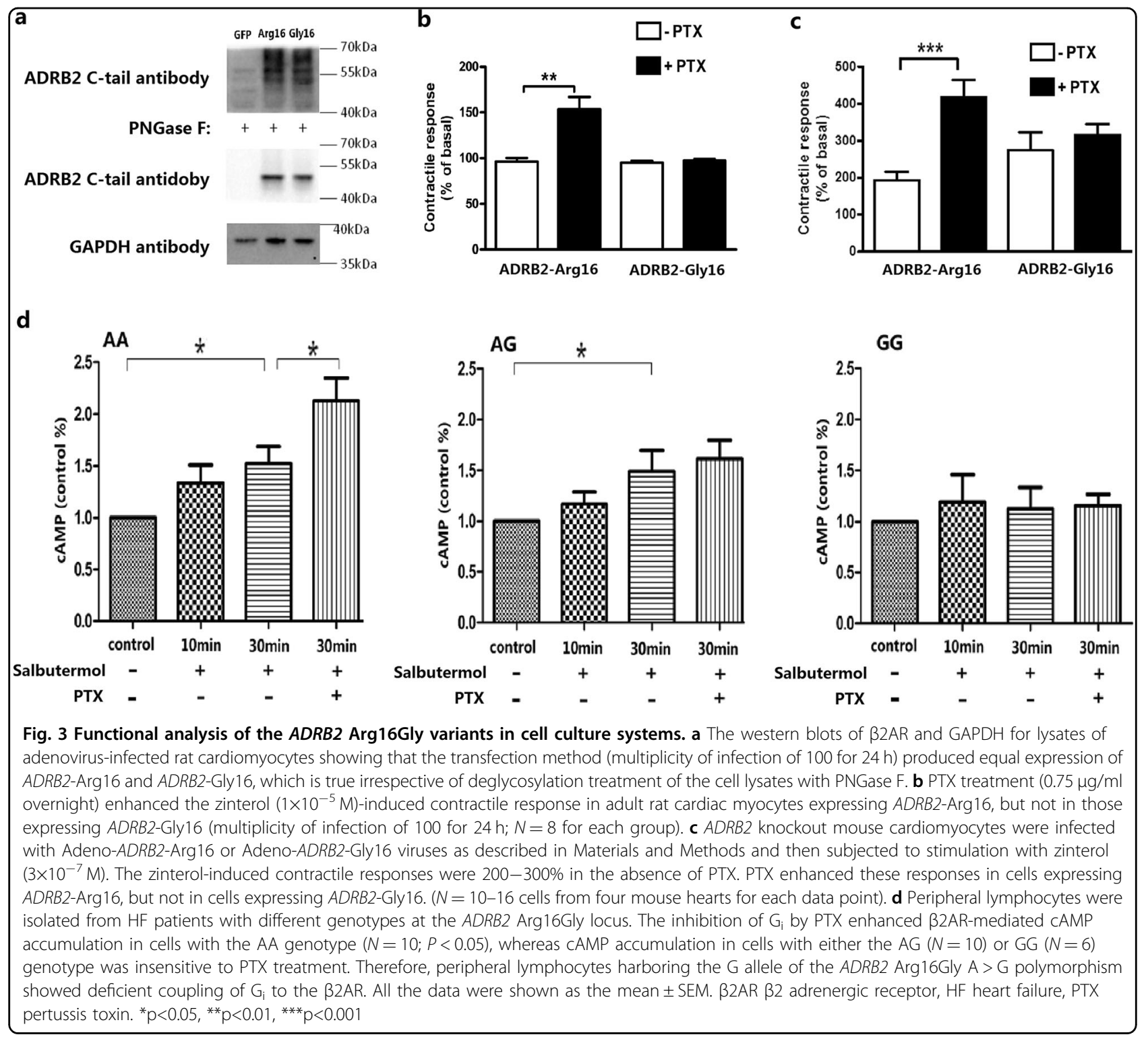

cohort. The influence of the G allele on the efficacy of BAR-blocker therapy is independent of conventional treatments, including angiotensin-converting enzyme inhibitors, spironolactone, digoxin, and diuretics with multiple adjustments. These findings provide a molecular basis among others for the long-standing question in the cardiovascular field of why nearly half of the HF patients do not respond well to $\beta$-blocker therapy ${ }^{24-27}$.

Mechanistically, we have demonstrated that disruption of $G_{i}$ signaling with PTX cannot alter $\beta 2 A R$-mediated contractile response in cardiomyocytes expressing $A D R B 2-G l y 16$, but markedly enhances the positive inotropic effect of $\beta 2 A R$ stimulation in cardiomyocytes expressing ADRB2-Arg16 in both adult rat model and B2AR-deficient mouse model (Fig. 3b, c). This conclusion is corroborated by the fact that PTX potentiates $\beta 2 \mathrm{AR}$ - stimulated cAMP production in human lymphocytes carrying two ADRB2-Arg16 alleles but not in cells expressing ADRB2-Gly16 (Fig. 3d). Taken together, we have demonstrated that $\beta 2$ ARs encoded by ADRB2-Gly16 cannot couple to $G_{i}$, while wild-type $\beta 2$ ARs (encoded by ADRB2-Arg16) activate both $\mathrm{G}_{\mathrm{s}}$ and $\mathrm{G}_{\mathrm{i}}$ signaling pathways.

In $\mathrm{HF}$, the hyperactivity of the sympathetic nervous system with an excessive $\beta A R$ stimulation-the salient feature of this syndrome-is widely considered to be a compensatory mechanism for impaired heart function $^{16,28}$. In the acute phase, activated $\beta A R$ signaling is beneficial to meet the demands of the body; however, chronic $\beta A R$ stimulation, especially $\beta 1 \mathrm{AR}$ persistent activation, initiates a cascade of cardiac hypertrophy and apoptosis via evoking CAMKII signaling pathway ${ }^{11}$. $\beta 1 \mathrm{AR}$ 
and $\beta 2 \mathrm{AR}$ are coexpressed in the heart, but exhibit distinct functions under certain pathological circumstances, such as chronic HF. Unlike the $\beta 1 A R$, which only couples to $G_{s}, \beta 2 A R$ also couples to PTX-sensitive $G_{i}$ proteins ${ }^{15}$ and mediates cardioprotective effects through the $G_{i}-G \beta \gamma-P I 3 K-A K T$ cell survival pathway ${ }^{18,19}$. Previous studies have shown that the deficiency of $\beta 2 A R$ enhances isoproterenol- or doxorubicin-induced myocardial injuries and mortality in mice $\mathrm{e}^{29,30}$, and the loss-of-function ADRB2 Thr164Ile mutation is associated with increased mortality in patients with $\mathrm{HF}^{31}$. These outcomes are likely the consequence of the loss of the $\beta 2 A R-$ $\mathrm{G}_{\mathrm{i}}-\mathrm{G} \beta \gamma-\mathrm{PI} 3 \mathrm{~K}-\mathrm{AKT}$ cell survival pathway ${ }^{18,19}$. Meanwhile, $\beta 2 A R-G i$ signaling has been reported to mediate the crosstalk of $\beta$ adrenergic receptor subtypes. Specifically, $\beta 2 A R-G_{i}$ signaling abrogates $\beta 1 A R$-induced loss of cardiomyocytes and negates both $\beta 1 \mathrm{AR}$-mediated and $\beta 2 A R$-mediated positive inotropic effects by negating the activation of 1-type calcium channel and CaMKII ${ }^{32-35}$. Thus, $\beta 2 A R$-evoked $G_{i}$ signaling biologically mimics the $\beta 1 \mathrm{AR}$ blocking effect. In the present study, we have demonstrated that $A D R B 2-G l y 16$ is deficient in its coupling to $G_{i}$ proteins (loss of function), which resulted in a loss of endogenous protection. In addition, the deficiency of the $\beta 2 A R-G_{i}$-signaling may exaggerate the pathogenic $\beta 1 \mathrm{AR}$ signaling. Thus, in the absence of $\beta A R$-blocker treatment, the subgroups of patients with AG or GG genotype have a markedly worse prognosis compared with patients with the AA genotype. Meanwhile, the reduced inhibition of the $\beta 1 A R-G_{s}$ signaling by the $\beta 2 A R-$ $\mathrm{G}_{\mathrm{i}}$ in ADRB2-Gly16-expressing myocardial cells may explain why the $G$ allele carriers are hypersensitive to $\beta A R$-blocker therapy. Moreover, we and others have demonstrated that selective activation of $\beta 2 A R$-coupled $G_{s}$ with fenoterol is also beneficial in multiple HF models $^{36,37}$, and that $\beta 2 A R$-mediated $G_{s}$ signaling provides contractile support without evoking the CaMKIIdependent cell death signaling cascade ${ }^{38}$. Thus, finely tuned $\beta 2 A R$-coupled $G_{s}$ and $G_{i}$ signaling has important pathological and therapeutic implications in HF. Especially for patients who are insensitive to $\beta 1 \mathrm{AR}$ blockade therapy, $\beta_{2}$-AR activation treating might be a potential novel therapy in future ${ }^{39}$.

Notably, several previous studies have been conducted to investigate the association between ADRB2 polymorphism and HF prognosis; however, the results are lack of enough power and not consistent ${ }^{31,40,41}$. The reason is mainly due to racial differences in the study populations. While exclusively Han Chinese are enrolled in the present study, the major composition of the study populations in these previous studies is Caucasian $(>70 \%)^{31,40,41}$. For the $A D R B 2 \operatorname{Arg} 16 \mathrm{Gly} \mathrm{A}>\mathrm{G}$ variant, the $\mathrm{G}$ allele is the minor allele in the Chinese Han population, with a frequency of $38.2 \%$ in our study, whereas it is the major allele in the European population (61.4\%, 1000 Genomes Project Phase 3: http://asia.ensembl.org/Homo_sapiens/ Variation/Population? $\mathrm{db}=$ core; $\mathrm{r}=5: 148826377-148827377$; $\mathrm{v}=\mathrm{rs} 1042713 ; \mathrm{vdb}=$ variation $; \mathrm{vf}=747520)^{42}$. Moreover, AA homozygotes (38.4\% in our study), which have poor response to $\beta$-blockers, exhibit only a frequency of $13.5 \%$ in the European population ${ }^{42}$. The nonresponsiveness to $\beta$-blocker therapy was not readily detected in previous studies, because those studies enrolled large proportions of Caucasians, which had a low frequency of AA homozygotes $^{31,40,41}$. In addition, small sample sizes (500 for one study and 186 for another) and limited follow-up period (12 months) in some studies also partly explain why previous studies have failed to reveal this association ${ }^{40,41}$. Differences in inclusion criteria may also contribute to the observed inconsistency between this and previous studies. For instance, most previous studies have only included HF patients with reduced ejection fraction $(<35-40 \%)^{20-22}$, whereas our study included both HFrEF and HFpEF subjects.

In addition to the $A D R B 2$ Arg16Gly variant, previous studies have shown the contributions of other naturally existing SNPs in related genes to the heterogeneity of HF patients to $\beta$-blocker therapy ${ }^{9,24-27,43}$. In particular, for the $A D R B 1$ Arg389Gly $\mathrm{C}>\mathrm{G}$ variant, we have shown that homozygotes ADRB1-Arg389 in our HF cohort exhibit a better response to $\beta$ AR-blocker treatment $(\mathrm{HR}, 0.52 ; P=$ 0.002) than Gly389 homozygotes (HR, $0.51 ; P=0.05$ ) (Fig. 2i), similar to the situation of Caucasians ${ }^{26}$. Furthermore, HF patients harboring one or two Glu27 alleles in the $A D R B 2$ Gln27Glu locus (the $\mathrm{G}$ allele) exhibit better responses to $\beta$-blockers than $\mathrm{Gln} 27$ (the $\mathrm{C}$ allele) homozygotes (HR, 0.25 for the GG genotype; HR, 0.36 for the CG genotype; and HR, 0.66 for the CC genotype) (Fig. 2i), consistent with previous reports ${ }^{41,43}$.

For the GRK5 Gln41Leu A > T locus, the $\mathrm{T}$ allele frequency is much higher in African American populations (32.6\%) than in Chinese $(1.1-1.3 \%)$ or Caucasians $(2.6 \%)$ populations $^{25}$. African American HF patients with the T allele exhibit a greater survival rate in the absence of $\beta$ blockers than those carrying the A allele, but they are resistant to $\beta$ AR-blocker therapy ${ }^{25,27}$. However, in our study, among 59 patients carrying the $\mathrm{T}$ allele, only three patients with and five patients without $\beta$ AR-blocker treatment experienced the primary end point event during follow-up. Thus, the present study is underpowered for this particular analysis.

Although clinical guidelines recommend maximal and nondiscriminatory prescription of $\beta$-blockers to all patients with stable HF in early stages, except for patients with comorbid contradictory conditions or who are unable to tolerate treatment with these drugs ${ }^{6,44}$, objective records indicate that only $57.2 \%$ of our patients $(64.3 \%$ of patients with NYHA class II HF, $56.3 \%$ of patients with 
NYHA class III HF, and $45.1 \%$ of patients with NYHA class IV HF) received $\beta$-blocker treatment. These medication rates have enabled us to collect comprehensive information regarding how patient genotype affects the progression of HF without the medication. Many previous studies, which have not demonstrated an effect of the $A D R B 2$ SNPs on HF prognosis, have medication rates of $80-100 \%$ for $\beta$-blockers ${ }^{45-48}$. Here, we are able to show on the basis of our data and previous reports that the therapeutic responses to $\beta$-blockers actually vary among genotypic subgroups of HF patients ${ }^{25-27}$. Application of $\beta$ AR-blocker therapy based on patient's genotype should be strongly recommended.

In conclusion, in this large-scale, multicenter and observational study of $\mathrm{HF}$, we have validated a strong association between the Arg16Gly A > G polymorphism in the $A D R B 2$ gene and clinical outcomes. The $\mathrm{G}$ allele carriers had a worse prognosis but responded well to $\beta$ blocker treatment. These findings provide both an explanation for the profound heterogeneity in the response to $\beta$-blocker therapy and the theoretical basis for individualizing $\beta$-blocker therapy in HF.

\section{Materials and methods}

\section{Study oversight}

The study was funded by the National Basic Research Program of China. The executive committee designed and oversaw the conduct of the study and data analysis. All protocols and methods were approved by the ethics committees of the local hospitals (Ethics Committee of Tongji Hospital) and conducted in accordance with the Declaration of Helsinki and the International Conference on Harmonization Guidelines for Good Clinical Practice. We obtained written informed consents from all participants. An independent data and safety monitoring committee periodically reviewed the study data.

\section{Study design}

A multicenter observational study was designed to investigate the combined effects of genotype and $\beta$ blocker therapy on prognosis, without interfering with any routine treatment procedures for patients with HF. In the preparatory stage, sequencing data for 100 pairs of HF patients and healthy control individuals from the local Chinese Han population were obtained to determine the allele frequency and distribution of polymorphisms of genes involved in $\beta A R$ signaling in this population. From January 2009, patients who were diagnosed as having HF were enrolled in this study at three centers in Southern (Tongji Hospital Affiliated to Tongji Medical Collage, Huazhong University of Science and Technology, Wuhan) and Northern (the Second Hospital of Hebei Medical University, Shijiazhuang; and Fuwai Hospital, National Center for Cardiovascular Disease, Beijing) China. A second separate cohort enrolled 919 patients with chronic HF and was used to validate the results observed in the first cohort. Patients were followed up periodically until the termination of the study. The primary end point was a composite of cardiovascular death or heart transplantation. The secondary end points included all-cause mortality, recurrence of HF, and first rehospitalization for cardiovascular causes. These rehospitalizations were defined as hospital admission for angina, arrhythmias, or HF-related symptoms, such as dyspnea or edema. Other related outcomes, such as hospitalization for any cause, new onset of stroke, and symptom relief as determined using the NYHA functional class, were also recorded during follow-up. The sample size and the number of required events were evaluated according to the preplanned interim analysis for the primary end point.

\section{Eligibility and study procedures}

From January 2009 to October 2014, 2615 patients were screened for eligibility for admission to the study. The replicated cohort included 919 patients with chronic HF recruited in Tongji Hospital (Wuhan) from October 2014 to March 2016. Eligibility requirements included age $\geq 18$ years, NYHA class II-IV HF with reduced or preserved left ventricular ejection fraction, and willingness to participate in long-term follow-up. The diagnosis of HF was confirmed by physical examination, laboratory tests, echocardiography, and according to the established protocols and criteria of the $\mathrm{ACC}$ and $\mathrm{AHA}^{6}$. At the time of study entry, detailed clinical data were obtained using a standardized questionnaire administered to the patients, with verification via medical records. The exclusion criteria were: severe valvular heart disease as the leading cause of HF; life-threatening complications, such as severe liver dysfunction, renal dysfunction, or a history of malignancy and life expectancy $<1$ year; second or third degree atrioventricular block, unless the patient had received a pacemaker; acute myocardial infarction or unstable angina within 1 month before admission; or refusal to participate in the follow-up. Whether the patient was treated with $\beta$-blockers was left to the treating physician's discretion. The investigators were instructed to make follow-up telephone calls or to perform face-toface interviews at predesignated times after patient discharge, to collect relevant information on the clinical events under evaluation. Information regarding medication usage for therapies administered for $>6$ months was also collected. The duration of follow-up was defined as the interval from the date of enrollment to the date when the primary end point occurred or the last contact.

\section{Resequencing and genotyping}

The DNA samples were prepared as previously reported $^{49}$. The coding regions of the ADRB1, ADRB2, GRK2, 
and GRK5 genes were screened via directional Sanger sequencing using the ABI 3130 BigDye Terminator v3.1 Cycle Sequencing Kit (Applied Biosystems, Foster City, CA, USA) on an ABI 3130xl sequencer. The primer sequences and amplicon sizes are given in Supplementary Table S12. The sequence analyses were performed using a 3130 Genetic Analyzer (Applied Biosystems, Foster City, CA, USA). The 7900 HT PCR system (Applied Biosystems, Foster City, CA, USA) and TaqMan allelic discrimination assay were used for genotyping. Forward and reverse TaqMan primers and FAM/VIC-labeled MGB probes, designed by Applied Biosystems or Shanghai GeneCore BioTechnologies Co. Ltd., were used for allelic discrimination (Supplementary Table S13), and the genotype assignments were performed using SDS Software v2.3.

\section{Adenoviruses}

Culture and adenovirus-mediated gene transfer of adult rat or mouse cardiomyocytes were implemented by methods described previously ${ }^{13,23}$. The starting DNA sequence for the generation of vector constructs of adenoviruses was that codes for the wild-type (Arg16) human $\beta 2 \mathrm{AR}$, with the following modifications: an $\mathrm{N}$-terminal hemagglutinin tag followed by a FLAG sequence, and a Cterminal polyhistidine $\operatorname{tag}^{50}$. The shuttle vectors were generated by sub-cloning. Site-directed mutagenesis on the $A D R B 2$ gene sequence was performed with the QuickChange II site-directed mutagenesis kit (Agilent Technologies, Santa Clara, CA, USA) to introduce a single nucleotide change (A to G, Arg16Gly) to mimic the naturally occurring polymorphism. An adenovirus carrying the wild-type human $A D R B 2-\operatorname{Arg} 16$ sequence, one carrying the minor allele sequence $A D R B 2-\mathrm{Gly} 16$, and the control adenovirus for jellyfish green fluorescent protein (GFP) were amplified, purified, and titered using established protocols described previously ${ }^{13,23}$.

\section{Cardiomyocyte isolation, adenoviral infection, and contractility measurement}

Cardiomyocytes were isolated from the hearts of Sprague-Dawley rats (male, 180-230 g) or ADRB2 knockout mice (male, 2-4 months) using standard enzymatic techniques ${ }^{13-15,23}$. Cardiomyocytes were seeded on laminin-coated coverslips and infected with the appropriate adenovirus at a multiplicity of infection of 100 . Subsequently, the myocytes were cultured for $24 \mathrm{~h}$ in medium supplemented with forskolin $(10 \mu \mathrm{M})$ and 2,3butanedione monoxime $(10 \mathrm{mM})$ to reestablish $\mathrm{G}_{\mathrm{i}}$ coupling of the $\beta 2 A R$ expressed from the viral vector ${ }^{23,51}$.

For protein determination, adenovirus-infected rat cardiomyocytes (about 50,000 cells) were lysed in a lysis buffer (Solarbio Science \& Technology, Beijing, China) containing a protease inhibitor mixture (Roche
Diagnostics, Basel, Switzerland). Cell lysates were clarified by centrifugation. A portion of the samples for $\beta 2 A R$ detection were further denatured and treated with peptide: $N$-glycosidase (PNGase F, New England Biolabs, Ipswich, MA, USA) for $2 \mathrm{~h}$ at $37^{\circ} \mathrm{C}$. The samples were denatured in Laemmli sample buffer and resolved by SDSPAGE. The $\beta 2 A R$ (C-tail antibody sc-569 from Santa Cruz Biotechnology, Santa Cruz, CA, USA, 1:1000) and GAPDH (antibody from EasyBio, China, 1:1000) were detected by immunoblotting. Densitometry was performed using NIH ImageJ. Ratios between the $\beta 2 \mathrm{AR}$ and the housekeeping protein GAPDH were calculated to normalize protein quantity.

Contractility measurements were performed as previously described ${ }^{15}$. Briefly, cardiomyocytes were perfused with a buffer containing $137 \mathrm{mM} \mathrm{NaCl}, 4.9 \mathrm{mM} \mathrm{KCl}$, $1.2 \mathrm{mM} \mathrm{MgCl}, 1 \mathrm{mM} \mathrm{NaH} \mathrm{PO}_{4}, 1 \mathrm{mM} \mathrm{CaCl}, 20 \mathrm{mM}$ glucose, and $20 \mathrm{mM}$ HEPES (pH 7.4), and electrically paced at ambient temperature on a microscopic stage. Cell length was monitored by an optical edge tracking method using an instrument setup manufactured by IonOptix (Milton, MA, USA). The measurements were made under steady-state conditions before and after treatment with a $\beta 2 \mathrm{AR}$ agonist, zinterol (from Tocris Bioscience, Bristol, UK; $10 \mu \mathrm{M}$ for rat cardiomyocytes or $0.3 \mu \mathrm{M}$ for $A D R B 2$ knockout mouse cardiomyocytes). In a subset of experiments, cells were pretreated with pertussis toxin (PTX, from List Biological Laboratories, Campbell, CA, USA; $0.75 \mu \mathrm{g} / \mathrm{ml}$ overnight) to block $G_{i}$ signaling, as previously described ${ }^{15,51}$.

\section{Human peripheral lymphocyte separation and measurement of cAMP accumulation}

Suspended lymphocytes from peripheral blood of HF patients with different genotypes of ADRB2 Arg16Gly site (AA, AG, or GG) (Supplementary Table S14) were prepared using lymphocyte separation medium (LTS10770125, Tian Jin Hao Yang Biological Manufacture, China) according to the manufacturer's instructions. The separated cells were seeded in six-well plates $\mathrm{V}$ $\left(10^{7}\right.$ cells/well) and cultured at $37^{\circ} \mathrm{C}$ in RPMI-1640 medium (Flow, Rockville, MD, USA) containing 10\% fetal bovine serum (Hyclone, Logan, UT, USA) and supplemented with $100 \mathrm{U} / \mathrm{ml}$ penicillin and $100 \mathrm{mg} / \mathrm{ml}$ streptomycin. $G_{i}$ signaling was specifically disrupted by pretreatment with $1.5 \mu \mathrm{g} / \mathrm{ml}$ PTX for $3 \mathrm{~h}$ before stimulation with $10 \mu \mathrm{M}$ salbutamol for 10 or $30 \mathrm{~min}$. To inhibit the degradation of cAMP, $200 \mu \mathrm{M}$ 3-isobutyl-1methylxanthine was added to each well $5 \mathrm{~min}$ before stopping the reaction at indicated time points. The cell lysates were used in the cAMP assay according to the instructions for the enzyme-linked immunosorbent assay kit (R\&D Systems, Minneapolis, MN, USA). The 
accumulation of cAMP in whole cell lysates was compared among different genotype groups.

\section{Statistical analysis}

We conducted a preplanned interim analysis for survival free from heart transplantation, and the sample size and required number of primary outcome events were calculated on the basis of the genotype of the ADRB2 Arg16Gly locus. Patients who were heterozygous or homozygous for Gly16 were combined into a single group, owing to the determined event rate. A 90\% power to detect significant prognostic differences between $\mathrm{G}$ allele carriers and the AA homozygotes of ADRB2 Arg16Gly polymorphism at an overall two-sided alpha level of 0.05 was desired. To achieve this outcome, with 344 effective events, we estimated that approximately 2700 patients would need to be followed up. However, the event rate was higher than expected. As of July 2015, 419 primary end points occurred in all patients enrolled in the first cohort, providing a power of $96 \%$ to detect a significant difference for this outcome. We therefore decided to terminate and selected July 2015 as the cutoff date for the final summary of the ADRB2 Arg16Gly variant analysis in the first cohort.

The clinical baseline characteristics of the participants enrolled in two cohorts are presented as the means, standard deviations; medians, interquartile range for continuous variables and counts and percentages for categorical variables. Comparisons between two groups were performed by independent samples $t$ test and chisquare test. Multiple comparisons were performed by one-way ANOVA using the post-hoc test. Time-to-event data were estimated using Kaplan-Meier analysis, and $P$ values were evaluated based on log-rank tests stratified according to the SNP genotypes. HRs and 95\% CIs for $\beta$ blocker treatment were estimated using Cox proportional hazards models. A two-sided $P$ value of 0.05 indicated statistical significance after adjustment for the clinical covariants with an unbalanced distribution between the groups. Improvement in NYHA functional class from enrollment through the follow-up period was analyzed as a binary outcome (improved condition versus no change or deteriorated condition) using a logistic regression model.

Statistical analyses were performed using the SPSS statistical software package (Version 16.0, SPSS Inc., Chicago, IL, USA) or Prism 5.0 (GraphPad Software Inc, San Diego, CA, USA). All reported $P$ values are two-sided, and those $<0.05$ were considered to be statistically significant.

\section{Acknowledgements}

This work was supported by the grants from National Program on Key Basic Research Project (973 Program) (No. 2012CB518004) and National Nature Science Foundation of China (No. 91439203, 81800356, and 81800336). We thank all the study researchers, as well as patients who participated in the present study.

\begin{abstract}
Author details
'Division of Cardiology, Departments of Internal Medicine, Tongji Hospital, Tongji Medical College, Huazhong University of Science \& Technology, 430030 Wuhan, China. ${ }^{2}$ Hubei Key Laboratory of Genetics and Molecular Mechanisms of Cardiologic Disorders, 430030 Wuhan, China. ${ }^{3}$ Institute of Molecular Medicine, Peking-Tsinghua Centre for Life Sciences, Peking University, 100871 Beijing, China. ${ }^{4}$ Fuwai Hospital, Chinese Academy of Medical Sciences and Peking Union Medical College, 100037 Beijing, China. ${ }^{5}$ Division of Cardiology, The Second Hospital of Hebei Medical University, 050000 Shijiazhuang, China. ${ }^{6}$ Department of Pharmacology, School of Life Sciences and Biopharmaceutics, Shenyang Pharmaceutical University, 110016 Shenyang, China
\end{abstract}

\section{Author contributions}

Corresponding authors were responsible for the study design, protocol development, study monitoring, and data management. Data analysis and manuscript drafting were performed by the first authors and guided by corresponding authors. All listed authors were involved in the study protocol review, data collection, and the decision to submit the manuscript for publication. Only the listed authors contributed to the writing of the manuscript. All authors assume responsibility for the accuracy and completeness of the data and for the fidelity of the study to the protocol.

Conflict of interest

The authors declare that they have no conflict of interest.

\section{Publisher's note}

Springer Nature remains neutral with regard to jurisdictional claims in published maps and institutional affiliations.

Supplementary Information accompanies the paper at (https://doi.org/ 10.1038/s41421-018-0058-6).

Received: 1 July 2018 Revised: 18 August 2018 Accepted: 20 August 2018 Published online: 23 October 2018

\section{References}

1. Mozaffarian, D. et al. Heart disease and stroke statistics - 2016 Update: a report from the American Heart Association. Circulation 133, e38-e360 (2016).

2. Jessup, M. \& Brozena, S. Heart failure. N. Engl. J. Med. 348, 2007-2018 (2003).

3. Braunwald, E. Biomarkers in heart failure. N. Engl. J. Med. 358, 2148-2159 (2008).

4. Barrese, V. \& Taglialatela, M. New advances in beta-blocker therapy in heart failure. Front. Physiol. 4, 323 (2013).

5. von Lueder, T. G. \& Krum, H. RAAS inhibitors and cardiovascular protection in large scale trials. Cardiovasc. Drugs Ther. 27, 171-179 (2013).

6. Hunt, S. A. et al. 2009 Focused update incorporated into the ACC/AHA 2005 Guidelines for the Diagnosis and Management of Heart Failure in Adults: a report of the American College of Cardiology Foundation/American Heart Association Task Force on Practice Guidelines developed in collaboration with the International Society for Heart and Lung Transplantation. J. Am. Coll. Cardiol. 53, e1-e90 (2009).

7. Liggett, S. B. Pharmacogenetic applications of the Human Genome project. Nat. Med. 7, 281-283 (2001).

8. Barsheshet, A., Brenyo, A., Goldenberg, I. \& Moss, A. J. Sex-related differences in patients' responses to heart failure therapy. Nat. Rev. Cardiol. 9, 234-242 (2012).

9. Talameh, J. A., McLeod, H. L., Adams, K. F.Jr. \& Patterson, J. H. Genetic tailoring of pharmacotherapy in heart failure: optimize the old, while we wait for something new. J. Card. Fail. 18, 338-349 (2012).

10. Shekelle, P. G. et al. Efficacy of angiotensin-converting enzyme inhibitors and beta-blockers in the management of left ventricular systolic dysfunction according to race, gender, and diabetic status: a meta-analysis of major clinical trials. J. Am. Coll. Cardiol. 41, 1529-1538 (2003). 
11. Yang, J., Liu, Y., Fan, X., Li, Z. \& Cheng, Y. A pathway and network review on beta-adrenoceptor signaling and beta blockers in cardiac remodeling. Heart Fail. Rev. 19, 799-814 (2014).

12. Zhang, R. et al. Calmodulin kinase II inhibition protects against structural heart disease. Nat. Med. 11, 409-417 (2005).

13. Zhu, W. Z. et al. Linkage of beta1-adrenergic stimulation to apoptotic heart cell death through protein kinase A-independent activation of $\mathrm{Ca} 2+/$ calmodulin kinase II. J. Clin. Invest. 111, 617-625, https://doi.org/10.1172/JCl16326 (2003).

14. Bristow, M. R. et al. Beta 1- and beta 2-adrenergic-receptor subpopulations in nonfailing and failing human ventricular myocardium: coupling of both receptor subtypes to muscle contraction and selective beta 1-receptor downregulation in heart failure. Circ. Res. 59, 297-309 (1986).

15. Xiao, R. P., Ji, X. \& Lakatta, E. G. Functional coupling of the beta 2-adrenoceptor to a pertussis toxin-sensitive $\mathrm{G}$ protein in cardiac myocytes. Mol. Pharmacol. 47, 322-329 (1995).

16. Rockman, H. A., Koch, W. J. \& Lefkowitz, R. J. Seven-transmembrane-spanning receptors and heart function. Nature 415, 206-212 (2002).

17. Feldman, A. M. et al. Increase of the $40,000-$ mol wt pertussis toxin substrate (G protein) in the failing human heart. J. Clin. Invest. 82, 189-197 (1988).

18. Chesley, A. et al. The beta(2)-adrenergic receptor delivers an antiapoptotic signal to cardiac myocytes through $\mathrm{G}(\mathrm{i})$-dependent coupling to phosphatidylinositol 3'-kinase. Circ. Res. 87, 1172-1179 (2000).

19. Zhu, W. Z. et al. Dual modulation of cell survival and cell death by beta(2)adrenergic signaling in adult mouse cardiac myocytes. Proc. Natl. Acad. Sci. USA 98, 1607-1612 (2001).

20. MERIT-HF Study Group. Effect of metoprolol CR/XL in chronic heart failure: Metoprolol CR/XL Randomised Intervention Trial in Congestive Heart Failure (MERIT-HF). Lancet 353, 2001-2007 (1999).

21. CIBIS-II Investigators and Committees. The Cardiac Insufficiency Bisoprolol Study II (CIBIS-II): a randomised trial. Lancet 353, 9-13 (1999).

22. The Beta-blocker Evaluation of Survival Trials (BEST) Investigators. Beta-Blocker Evaluation of Survival Trial, I. A trial of the beta-blocker bucindolol in patients with advanced chronic heart failure. N. Engl. J. Med. 344, 1659-1667 (2001).

23. Zhou, Y. Y. et al. Culture and adenoviral infection of adult mouse cardiac myocytes: methods for cellular genetic physiology. Am. J. Physiol. Heart Circ. Physiol. 279, H429-H436 (2000).

24. Mialet Perez, J. et al. Beta 1-adrenergic receptor polymorphisms confer differential function and predisposition to heart failure. Nat. Med. 9, 1300-1305 (2003).

25. Liggett, S. B. et al. A GRK5 polymorphism that inhibits beta-adrenergic receptor signaling is protective in heart failure. Nat. Med. 14, 510-517 (2008).

26. Liggett, S. B. et al. A polymorphism within a conserved beta(1)-adrenergic receptor motif alters cardiac function and beta-blocker response in human heart failure. Proc. Natl Acad. Sci. USA 103, 11288-11293 (2006).

27. Cresci, S. et al. Clinical and genetic modifiers of long-term survival in heart failure. J. Am. Coll. Cardiol. 54, 432-444 (2009).

28. Port, J. D. \& Bristow, M. R. Altered beta-adrenergic receptor gene regulation and signaling in chronic heart failure. J. Mol. Cell. Cardiol. 33, 887-905 (2001).

29. Bernstein, D. et al. Differential cardioprotective/cardiotoxic effects mediated by beta-adrenergic receptor subtypes. Am. J. Physiol. Heart Circ. Physiol. 289, H2441-H2449 (2005).

30. Patterson, A. J. et al. Protecting the myocardium: a role for the beta2 adrenergic receptor in the heart. Crit. Care Med. 32, 1041-1048 (2004).

31. Liggett, S. B. et al. The lle164 beta2-adrenergic receptor polymorphism adversely affects the outcome of congestive heart failure. J. Clin. Invest. 102 1534-1539 (1998).
32. Zhu, W., Zeng, X., Zheng, M. \& Xiao, R. P. The enigma of beta2-adrenergic receptor Gi signaling in the heart: the good, the bad, and the ugly. Circ. Res. 97, 507-509 (2005).

33. Sato, M., Gong, H., Terracciano, C. M., Ranu, H. \& Harding, S. E. Loss of betaadrenoceptorresponse in myocytes overexpressing the $\mathrm{Na}+/ \mathrm{Ca}(2+)$ -exchanger. J. Mol. Cell. Cardiol. 36, 43-48 (2004)

34. He, J. Q., Balijepalli, R. C., Haworth, R. A. \& Kamp, T. J. Crosstalk of betaadrenergic receptor subtypes through $\mathrm{Gi}$ blunts beta-adrenergic stimulation of L-type Ca2+channels in canine heart failure. Circ. Res. 97, 566-573 (2005).

35. Xiao, R. P. \& Balke, C. W. Na+/Ca2+exchange linking beta2-adrenergic G(i) signaling to heart failure: associated defect of adrenergic contractile support. J. Mol. Cell. Cardiol. 36, 7-11 (2004)

36. Ahmet, l. et al. Beneficial effects of chronic pharmacological manipulation of beta-adrenoreceptor subtype signaling in rodent dilated ischemic cardiomyopathy. Circulation 110, 1083-1090 (2004).

37. Chakir, K. et al. Galphas-biased beta2-adrenergic receptor signaling from restoring synchronous contraction in the failing heart. Sci. Transl. Med 3, 100ra188 (2011).

38. Mangmool, S., Shukla, A. K. \& Rockman, H. A. beta-Arrestin-dependent activation of $\mathrm{Ca}(2+) /$ calmodulin kinase $\|$ after beta(1)-adrenergic receptor stimulation. J. Cell. Biol. 189, 573-587 (2010).

39. Kim, K. E. et al. Cardiac physiologic regulation of sub-type specific adrenergic receptors in transgenic mice overexpressing beta1- and beta2-adrenergic receptors. Clin. Exp. Emerg. Med. 3, 175-180 (2016)

40. Mansur, A. J. et al. Beta-2 adrenergic receptor gene polymorphisms GIn27Glu, Arg16Gly in patients with heart failure. BMC Cardiovasc. Disord. 9, 50 (2009).

41. Metra, M. et al. Role of beta-adrenergic receptor gene polymorphisms in the long-term effects of beta-blockade with carvedilol in patients with chronic heart failure. Cardiovasc. Drugs Ther. 24, 49-60 (2010).

42. Genomes Project, $C$. et al. A global reference for human genetic variation Nature 526, 68-74 (2015).

43. Kaye, D. M. et al. Beta-adrenoceptor genotype influences the response to carvedilol in patients with congestive heart failure. Pharmacogenetics $\mathbf{1 3}$ 379-382 (2003).

44. Taylor, J. The 2012 ESC Guidelines on Heart Failure. Eur. Heart J. 33, 1703-1704 (2012).

45. de Groote, P. et al. The impact of beta-adrenoreceptor gene polymorphisms on survival in patients with congestive heart failure. Eur. J. Heart Fail. 7 966-973 (2005).

46. Leineweber, $\mathrm{K}$. et al. The Arg16Gly-beta(2)-adrenoceptor single nucleotide polymorphism: exercise capacity and survival in patients with end-stage heart failure. Naunyn Schmiede. Arch. Pharmacol. 382, 357-365 (2010).

47. Sehnert, A. J. et al. Lack of association between adrenergic receptor genotypes and survival in heart failure patients treated with carvedilol or metoprolol. J. Am. Coll. Cardiol. 52, 644-651 (2008).

48. Shin, J. et al. Relation of beta(2)-adrenoceptor haplotype to risk of death and heart transplantation in patients with heart failure. Am. J. Cardiol. 99, 250-255 (2007).

49. Zhang, L. et al. Comparison of high-resolution melting analysis, TaqMan Allelic discrimination assay, and sanger sequencing for Clopidogrel efficacy genotyping in routine molecular diagnosticsJ. Mol. Diagn. 15, 600-606 (2013).

50. Kobilka, B. K. Amino and carboxyl terminal modifications to facilitate the production and purification of a $\mathrm{G}$ protein-coupled receptor. Anal. Biochem. 231, 269-271 (1995)

51. Woo, A. Y. et al. Tyrosine 308 is necessary for ligand-directed Gs protein-biased signaling of beta2-adrenoceptor. J. Biol. Chem. 289, 19351-19363 (2014). 